\title{
La investigación pedagógica en la Escuela de Pedagogía de la UPS Sede Quito
}

\author{
Comisión de Investigaciones Pedagógicas*
}

\section{Las tesis de grado}

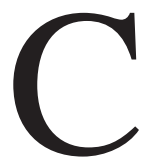

omo primera parte del estudio sobre un análisis de las investigaciones pedagógicas, con la finalidad de elaborar una propuesta para la programación de las investigaciones en la Facultad, hemos comenzado por un diagnóstico interpretativo y explicativo de los procesos de investigación a partir de las tesis de grado: sus campos de estudios o problemas abordados, las metodologías empleadas, las orientaciones institucionales y educativas, las áreas de estudio privilegiadas y la dirección de las tesis.

El objetivo de esta primera parte de la indagación consiste en destacar los procesos, procedimientos, recursos académicos, los intereses y presupuestos ideológicos, que orientaron las investigaciones y tesis durante la historia de lo que ha sido, en la UPS, la conformación y consolidación de la Facultad de Ciencias Humanas y de la Educación (FACHED).

Se pudieron diferenciar claramente dos épocas: la de formación académica, cuando los estudios pedagógicos eran parte de otros espacios universitarios, y la de consolidación académica, cuando se define un espacio institucional propio y específico dentro de la Universidad, para las especialidades relacionadas con la educación y la pedagogía propiamente dicha. Estas dos épocas han estado marcadas por la indefinición epistemólogica, propia de la pedagogía, la cual fue, por mucho

\footnotetext{
* La Comisión de Investigaciones Pedagógicas de la Universidad Politécnica Salesiana, autora del estudio, está integrada por María Helena Ortiz, Sebastián Granda, Jaime Padilla y coordinada por José Sánchez-Parga.
} 
tiempo, parte complementaria de las otras ciencias, como la filosofía o la psicología. Sin embargo, se debe destacar que el aparecimiento de la carrera de pedagogía, parvularia y EGB permitieron que las temáticas investigativas vayan tomando matices educativos más específicos.

Las razones para iniciar el estudio desde las tesis es porque precisamente en esta práctica académica terminal en la formación de los estudiantes, se manifiesta su nivel científico y académico e incluso la orientación de su formación profesional; también en las tesis se expresan en cierta manera las competencias e intereses investigativos y científicos, académicos y profesionales de los docentes directores de las tesis; finalmente, también las tesis reflejan la organización del pensum y programa de la carrera, la orientación curricular de sus materias y hasta los perfiles académicos y profesionales de los estudiantes.

De hecho la propuesta de una Programación de Investigaciones Educativas en la Universidad y en las Carreras o Facultad de Pedagogía habrá de tener en cuenta no solo todos los elementos que componen y organización el programa de estudio, sino también las orientaciones científicas y académicas de la misma carrera así como la formación profesional de los estudiantes, y finalmente los procedimientos referentes a la dirección y elaboración de las investigaciones y tesis.

Finalmente hay que considerar que si bien la investigación habrá de desarrollarse a partir del trabajo de las tesis, donde converge la actividad docente y del estudiante, el desarrollo de un programa de investigaciones deberá trascender las prácticas de las tesis para ampliarse hacia otras prácticas.

\section{Evolución periódica de la tesis ${ }^{1}$}

Entre 1965 y 1994 durante tres décadas la evolución del número de tesis presentadas refleja un comportamiento extremadamente irregular de acuerdo a sucesivas épocas: entre 1965 y 1971 solo una o dos

\footnotetext{
'En el anexo \# 1 consta una breve descripción del desarrollo de la estructura académica del Área de Pedagogía. Esta información es fundamental para comprender los resultados y datos que se exponen a continuación. En el anexo \# 2 se puede encontrar todos los datos generados por el equipo de investigación en estos primeros cuatro meses de trabajo.
} 
tesis anuales; entre 1972-76 el número de tesis por año es en promedio superior a 10; vuelve a declinar durante las dos décadas siguientes (1977-94); y comienza a crecer de manera constante y regular desde 1996, cuando la carrera de Pedagogía adquiere su estatuto académico definitivo dentro de la Facultad de Ciencias Humanas.

Es importante notar cómo la actividad académica de la investigación en las tesis de licenciatura alcanza una relativa estabilidad a medida que la misma carrera queda institucionalizada en el marco universitario de una Facultad y sujeta a una cierta normalización de sus procesos académicos. Incluso las investigaciones / tesis adquieren una mayor especificidad científica, centrándose de manera preferente en el campo de la pedagogía y procesos educativos.

De todas maneras será necesario explicar las relativas alteraciones en el número de tesis presentadas, su frecuencia, que tienen lugar entre 1995 y 2003 . Además de la evolución y oscilaciones del número de los estudiantes matriculados en dicho período, habrá que contar con las tesis procedentes del programa Cotopaxi, que se inicia también en dicha época (1995-96), y del programa de profesionalización (1997); y cuya incidencia justificaría, por ejemplo, el crecimiento de tesis presentadas en los años 1999-2000 (62 y 66 respectivamente).

Un factor adicional sobre la evolución e irregularidades del número de tesis presentadas puede ser atribuido a la misma dirección de tesis, ya que de acuerdo a las entrevistas con docentes tanto la dedicación como la exigencia en la dirección de las investigaciones suele ser una garantía no solo para la conclusión de la tesis sino también para sus plazos.

En conclusión y de manera general todos los aspectos analizados (evolución periódica de la tesis, dirección de tesis, temas y campos de investigación, niveles educativos investigados, tipos de áreas y régimen de planteles educativos cubiertos por las investigaciones) coinciden en identificar dos épocas, que corresponden las dos fases de formación y consolidación académica de la carrera: 1965-1994 y 1995-2003. Lo que prueba la influencia de la organización y de las estructuras académicas en esta práctica tan importante y decisiva para la formación del estudiante y el mismo desarrollo académicos como son las investigaciones finales de la carrera. 


\section{Dirección de tesis}

91 profesores entre el año 1965 y 2003 dirigieron 475 tesis, y en 17 tesis no constan los nombres de los profesores que las dirigieron. Llama poderosamente la atención que un gran número de profesores (34) solo hayan dirigido una tesis, y que un número muy elevado de tesis hayan sido dirigidas por un solo profesor. Lo que pone de manifiesto una falta de política y programación académicas en esta práctica de la dirección de tesis.

\section{Número de directores de tesis por número de tesis}

A esta situación hay que añadir el excesivo número de profesores que han dirigido tesis cada año, lo que supone una enorme dispersión de esta actividad académica, la cual debería concentrarse en base a algunos criterios: mayores competencias de algunos docentes para la dirección de las tesis; áreas o campos de conocimientos definidos en los programas y cubiertos por determinado número de docentes; orientación de la carrera y de los perfiles profesionales. Una tal dispersión en la dirección de tesis impide tanto la especialización de los docentes en esta actividad académica como la formación de una masa crítica resultado de dicha dirección de tesis. La dirección de la tesis podría reagrupar estudiantes que investigan un mismo campo de la ciencia o área homogénea de conocimientos.

\begin{tabular}{|c|c|c|c|c|}
\hline N. Tesis & N. Direct & \% Direct & Total Tes & $\%$ Tesis \\
\hline 1 & 34 & 37,4 & 34 & 7,2 \\
\hline 2 & 13 & 14,3 & 26 & 5,5 \\
\hline 3 & 10 & 11 & 30 & 6,3 \\
\hline 4 & 7 & 7,69 & 28 & 5,9 \\
\hline 5 & 5 & 5,49 & 25 & 5,3 \\
\hline 6 & 4 & 4,4 & 24 & 5,1 \\
\hline 7 & 2 & 2,2 & 14 & 2,9 \\
\hline 8 & 3 & 3,3 & 24 & 5,1 \\
\hline 9 & 2 & 2,2 & 18 & 3,8 \\
\hline
\end{tabular}




\begin{tabular}{|c|c|c|c|c|}
\hline 11 & 1 & 1,1 & 11 & 2,3 \\
\hline 13 & 1 & 1,1 & 13 & 2,7 \\
\hline 14 & 2 & 2,2 & 28 & 5,9 \\
\hline 17 & 2 & 2,2 & 34 & 7,2 \\
\hline 20 & 1 & 1,1 & 20 & 4,2 \\
\hline 30 & 1 & 1,1 & 30 & 6,3 \\
\hline 35 & 1 & 1,1 & 35 & 7,4 \\
\hline 38 & 1 & 1,1 & 38 & 8 \\
\hline 43 & 1 & 1,1 & 43 & 9,1 \\
\hline Total & 91 & 100 & 475 & 100 \\
\hline
\end{tabular}

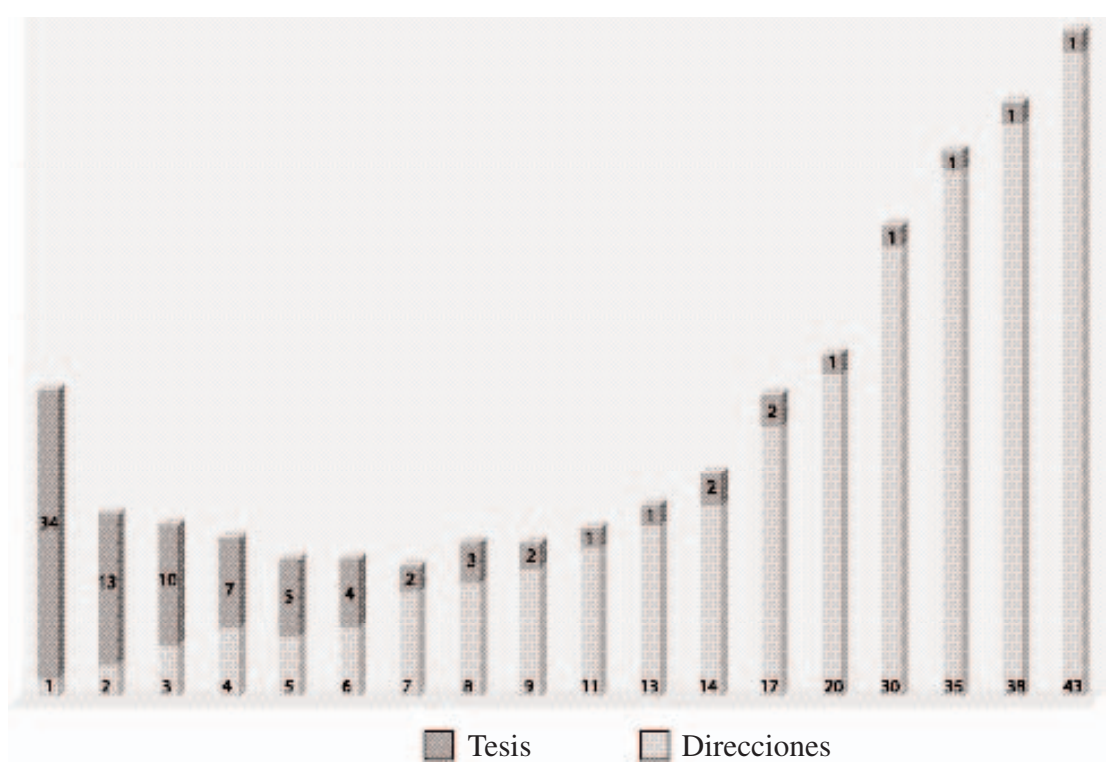

Número de Directores de Tesis por Año

Años 1965 - 1994

\begin{tabular}{|l|c|c|c|c|c|c|c|c|c|c|c|c|c|}
\hline Años & $\mathbf{6 9}$ & $\mathbf{7 0}$ & $\mathbf{7 1}$ & $\mathbf{7 2}$ & $\mathbf{7 3}$ & $\mathbf{7 4}$ & $\mathbf{7 5}$ & $\mathbf{7 6}$ & $\mathbf{7 7}$ & $\mathbf{7 8}$ & $\mathbf{7 9}$ & $\mathbf{8 0}$ & $\mathbf{8 1}$ \\
\hline Tesis & 2 & 2 & 1 & 11 & 10 & 6 & 2 & 12 & 4 & 1 & 4 & 3 & 2 \\
\hline Directores & 2 & 2 & 1 & 4 & 5 & 3 & 2 & 4 & 3 & 1 & 4 & 3 & 2 \\
\hline Promedio & $\mathbf{1}$ & $\mathbf{1}$ & $\mathbf{1}$ & $\mathbf{2 , 7}$ & $\mathbf{2}$ & $\mathbf{2}$ & $\mathbf{1}$ & $\mathbf{3}$ & $\mathbf{1 , 3}$ & $\mathbf{1}$ & $\mathbf{1}$ & $\mathbf{1}$ & $\mathbf{1}$ \\
\hline
\end{tabular}




\begin{tabular}{|l|c|c|c|c|c|c|c|c|c|c|c|c|c|}
\hline Años & $\mathbf{8 2}$ & $\mathbf{8 3}$ & $\mathbf{8 4}$ & $\mathbf{8 5}$ & $\mathbf{8 6}$ & $\mathbf{8 7}$ & $\mathbf{8 8}$ & $\mathbf{8 9}$ & $\mathbf{9 0}$ & $\mathbf{9 1}$ & $\mathbf{9 2}$ & $\mathbf{9 3}$ & $\mathbf{9 4}$ \\
\hline Tesis & 7 & 6 & 5 & 4 & 2 & 2 & 2 & 2 & 3 & 2 & 7 & 7 & 8 \\
\hline Directores & 2 & 5 & 3 & 3 & 2 & 2 & 2 & 2 & 2 & 2 & 4 & 4 & 5 \\
\hline Promedio & $\mathbf{3 , 5}$ & $\mathbf{1 , 2}$ & $\mathbf{1 , 7}$ & $\mathbf{1 , 3}$ & $\mathbf{1}$ & $\mathbf{1}$ & $\mathbf{1}$ & $\mathbf{1}$ & $\mathbf{1 , 5}$ & $\mathbf{1}$ & $\mathbf{1 , 8}$ & $\mathbf{1 , 8}$ & $\mathbf{1 , 6}$ \\
\hline
\end{tabular}

Años 1995 - 2003

\begin{tabular}{|l|c|c|c|c|c|c|c|c|c|c|}
\hline Años & $\mathbf{1 9 9 5}$ & $\mathbf{1 9 9 6}$ & $\mathbf{1 9 9 7}$ & $\mathbf{1 9 9 8}$ & $\mathbf{1 9 9 9}$ & $\mathbf{2 0 0 0}$ & $\mathbf{2 0 0 1}$ & $\mathbf{2 0 0 2}$ & $\mathbf{2 0 0 3}$ & S/f \\
\hline Tesis & 7 & 14 & 45 & 32 & 60 & 65 & 35 & 45 & 52 & 3 \\
\hline Directores & 5 & 12 & 14 & 13 & 25 & 25 & 16 & 23 & 23 & 3 \\
\hline Promedio & $\mathbf{1 , 4}$ & $\mathbf{1 , 2}$ & $\mathbf{3 , 2}$ & $\mathbf{2 , 5}$ & $\mathbf{2 , 4}$ & $\mathbf{2 , 6}$ & $\mathbf{2 , 2}$ & $\mathbf{2}$ & $\mathbf{2 , 3}$ & $\mathbf{1}$ \\
\hline
\end{tabular}

En el período de 1965-1994 han sido 7 profesores salesianos los que han dirigido más de la mitad de las tesis, el $68,4 \%$ (80 sobre un total de 117).

Esta información puede completarse por el excesivo número de profesores que han dirigido tesis en un reducido número de años, frente al reducido número de profesores que han dirigido tesis en mayor número de años durante el período de 1995-2003.

\begin{tabular}{|c|c|}
\hline Nro. Profesores & En dirección Tesis \\
\hline 10 & 1 año \\
\hline 6 & 2 años \\
\hline 2 & 3 años \\
\hline 2 & 5 años \\
\hline 2 & 6 años \\
\hline 1 & 4 años \\
\hline
\end{tabular}

Otro dato importante es la especialidad y títulos académicos o profesión de los directores de las tesis. De 35 de los directores no consta información alguna sobre su título o especialidad académica; de 20 se señala que "ya no trabaja" y por consiguiente tampoco de ellos hay información académica; de los 13 salesianos tampoco consta la referencia académica. 
Respecto de las especializaciones registradas hay 5 sociólogos, 5 psicólogos, 5 teólogos, 3 filósofos, 2 lingüistas, 1 ingeniero comercial, 1 antropólogo, 1 médico, 1 comunicador, 1 biólogo y 1 abogado. Este dato explica en cierto modo la diversidad de orientaciones que han tenidos muchas de las tesis dirigidas, en pedagogía.

De un total de los docentes directores de tesis el $70 \%$ no tienen más título que el de licenciado, y del 30\% con títulos superiores de postgrado, un $90 \%$ no posee postgrado en pedagogía.

Otro aspecto cabe resaltar respecto de la inestabilidad académica de los docentes directores de tesis. Solo 15 docentes directores de tesis entre 1992 y 1997 permanecen en la UPS y únicamente 22 directores de tesis entre 1998-2003 siguen de docentes en la UPS. Es decir menos del $30 \%$ de directores de tesis siguen como docentes en la UPS.

Finalmente hay que destacar una de las situaciones que merecen mayor organización y programación académicas en el futuro: la dispersión de directores de tesis y la dispersión de temas. Debiéndose evitar que sean muchos profesores quienes dirigen tesis sobre un mismo tema o campo de conocimientos, y que un mismo profesor dirija tesis sobre temas o campos de conocimientos muy diferentes.

\section{Objetos o problemas de investigación de las tesis}

Un análisis detallado de las tesis realizadas entre 1965 y 2003 muestra tres fenómenos singulares respecto a los objetos o problemas de investigación de las tesis.

a) La extraordinaria diversidad de temas y problemáticas investigadas. A lo largo del período analizado se han investigado un total de 39 temas diferentes, sin contar aquellas tesis cuyos temas no pudieron ser registrados con los indicadores diseñados por el equipo.

\begin{tabular}{|l|r|r|r|}
\hline Temas & ISS & \multicolumn{1}{|c|}{ UPS } & Total \\
\hline Aprendizaje & 0 & 12 & $\mathbf{1 2}$ \\
\hline Fact.Pedagóg.Aprendizaje & 1 & 21 & $\mathbf{2 2}$ \\
\hline Fact.Psicológ.Aprendizaje & 0 & 10 & $\mathbf{1 0}$ \\
\hline Fact.Sociales Aprendizaje & 1 & 5 & $\mathbf{6}$ \\
\hline
\end{tabular}




\begin{tabular}{|c|c|c|c|}
\hline Temas & ISS & UPS & Total \\
\hline Problemas de aprendizaje & 0 & 7 & 7 \\
\hline Rendimiento & 0 & 4 & 4 \\
\hline Fact.Pedagóg.Rendimiento & 0 & 30 & 30 \\
\hline Fact.Psicológ.Rendimiento & 1 & 7 & 8 \\
\hline Fact.Sociales Rendimiento & 0 & 20 & 20 \\
\hline Afectividad & 0 & 2 & 2 \\
\hline Autoestima & 0 & 2 & 2 \\
\hline Comportamiento & 3 & 1 & 4 \\
\hline Fact.Pedagóg.Comportam. & 0 & 2 & 2 \\
\hline Fact.Sociales Comportam. & 2 & 1 & 3 \\
\hline Catequesis & 5 & 0 & 5 \\
\hline Deserción & 0 & 8 & 8 \\
\hline Educación ambiental & 0 & 1 & 1 \\
\hline Enseñanza & 0 & 5 & 5 \\
\hline Estimulación temprana & 0 & 2 & 2 \\
\hline Etnografía & 1 & 7 & 8 \\
\hline Evaluación & 2 & 13 & 15 \\
\hline Fact.Psicológ.Inteligencia & 0 & 1 & 1 \\
\hline Fact.Sociales Inteligencia & 1 & 1 & 2 \\
\hline Fact.Sociales Personalid. & 1 & 0 & 1 \\
\hline Filosofía & 11 & 11 & 22 \\
\hline Filosofía de la Educación & 10 & 10 & 20 \\
\hline Género & 0 & 4 & 4 \\
\hline Integración escolar & 0 & 1 & 1 \\
\hline Intervención Psicomotriz & 0 & 1 & $\mathbf{1}$ \\
\hline Metodología & 17 & 82 & 99 \\
\hline Migración & 0 & 1 & 1 \\
\hline Orientación Vocacional & 17 & 19 & 36 \\
\hline Psicología & 5 & 3 & 8 \\
\hline Planificación Familiar & 1 & 0 & 1 \\
\hline Religión & 17 & 7 & 24 \\
\hline Repitencia & 0 & 6 & 6 \\
\hline Sistema Educativo & 6 & 13 & 19 \\
\hline Test & 15 & 4 & 19 \\
\hline Valores y derechos & 3 & 13 & 16 \\
\hline Otros & 2 & 31 & 33 \\
\hline $\mathrm{N}^{0}$ Tesis por año & 122 & 368 & 490 \\
\hline
\end{tabular}


b) La numerosa cantidad de tesis cuyos objetos de investigación nada o casi nada tienen que ver con la pedagogía y la educación. De todas las tesis realizadas en el período estudiado, 238 tesis (56.8\%) investigan problemas educativos y pedagógicos (rendimiento, aprendizaje, evaluación, etc.), mientras que 181 (43.2\%) tienen por objeto otros temas: religión, catequesis, planificación familiar, entre otros.

c) El alto porcentaje de tesis que analizan problemáticas pedagógicas y educativas desde enfoques más psicológicos, sociológicos o filosóficos que específicamente pedagógicos. Es el caso, por ejemplo, de las tesis cuyo objeto de investigación ha sido el "rendimiento": de un total de 57 tesis que analizan el tema del rendimiento, 20 (35\%) lo hacen desde un enfoque sociológico y 7 (12.2\%) desde una perspectiva psicológica.

\begin{tabular}{|c|c|}
\hline Nro. de tesis sobre rendimiento & Enfoque de estudio \\
\hline 30 & pedagógico \\
\hline 7 & psicológico \\
\hline 20 & sociológico \\
\hline
\end{tabular}

Si realizamos un análisis más de corte histórico, constatamos que hay temas que han sido investigados a lo largo de todo el período analizado, como es el caso de "filosofía", "orientación vocacional" y "religión"; mientras que otros temas han sido investigados durante períodos cortos: los "factores pedagógicos del aprendizaje" han sido objeto de investigación casi exclusivamente en el período 1996 - 2003 (21 de 22 tesis).

En todo caso, se evidencia que los temas de tesis se encuentran muy condicionados por el desarrollo de las carreras y su institucionalización académica. Así, por ejemplo, el hecho que la mayor parte de tesis sobre "factores pedagógicos del aprendizaje" se hayan desarrollado en el período 1996 - 2003, se explica porque en ese período se encontraban en pleno funcionamiento las carreras de pedagogía, docencia básica intercultural bilingüe, así como el programa de profesionalización. 


\section{Metodología de las investigaciones}

Uno de los datos más singulares y también más reveladores de la orientación y las características de las investigaciones y tesis realizadas tiene que ver con las metodologías empleadas. Mientras que el ensayo, que no constituye propiamente una investigación, es el género más frecuente en el período 1965-94 con 63 tesis (51.6\%), para reducirse considerablemente en el período 1995-2003 con 31 tesis (8.4\%), lo que se ha convenido en denominar "investigación de campo" casi se duplica en el transcurso de ambos períodos: de 59 (48.4\%) en 1965-94 pasa a $291(79.1 \%)$ en 1995-2003.

\begin{tabular}{|l|l|l|l|}
\hline \multicolumn{1}{|c|}{ Tipo de documento } & ISS & UPS & Total \\
\hline Ensayo & 63 & 31 & $\mathbf{9 4}$ \\
\hline Investigación de Campo & 59 & 291 & $\mathbf{3 5 0}$ \\
\hline Producto Educativo & 0 & 46 & $\mathbf{4 6}$ \\
\hline Total por época & $\mathbf{1 2 2}$ & $\mathbf{3 6 8}$ & $\mathbf{4 9 0}$ \\
\hline
\end{tabular}

SIMBOLOGÍA: Instituto Superior Salesiano (ISS) -Universidad Politécnica Salesiana

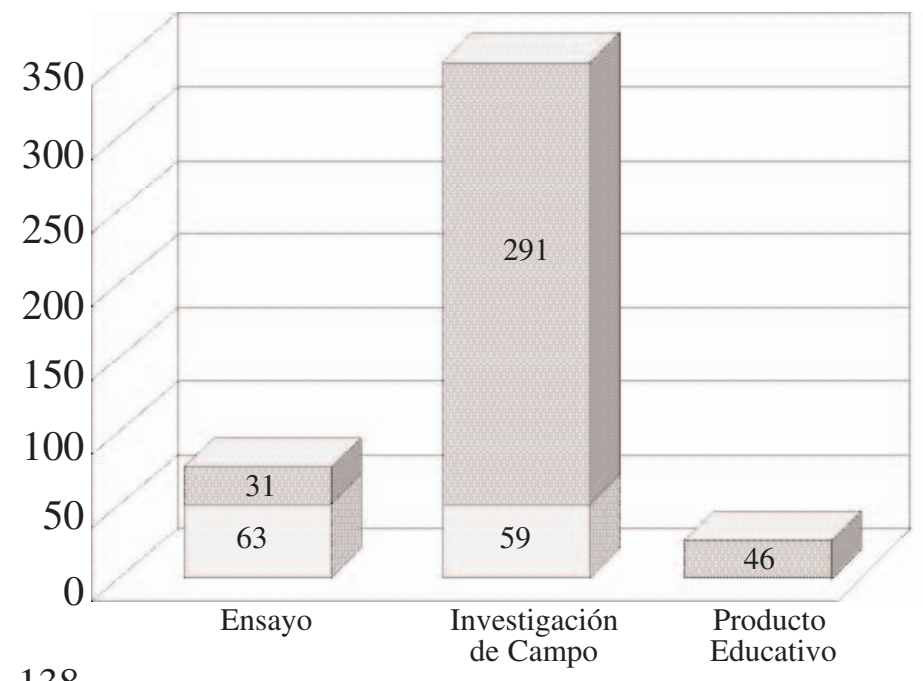


Bajo la categoría de "investigación de Campo" el mayor porcentaje de investigaciones se han realizado en base a un uso de métodos y técnicas más específicamente sociológicos que pedagógicos: encuestas y entrevistas, registros de datos estadísticos y de archivo. También se ha recurrido al método de carácter etnográfico.

Hay que notar sin embargo que el género de ensayo no desaparece como práctica académica en la presentación de las tesis. Otro dato interesante es la aparición de los llamados "productos educativos" durante el último período de 1995-2003 con 46 tesis (12.5\%), inexistente en el período anterior (1965-1994).

Tanto el "ensayo" como el "producto educativo" son dos prácticas académicas que no responden propiamente a una investigación, por razones ideológicas en un caso y por razones instrumentales en otro caso. Pero ambos fenómenos se explican en cierto modo por una indefinición académica y profesional en la dirección de las tesis, pero sobre todo por desconocer la específica importancia de la investigación en la formación de los estudiantes en cuanto ejercicio terminal de su carrera. En este sentido será necesario evaluar también el valor científico y académico de los "productos educativos" en cuanto objeto de tesis.

\section{Nivel educativo o campos de la educación investigados}

Mientras que en el período de 1965-1994 48 tesis (39.3\%) son consagradas al Ciclo Básico / Secundaria y Bachillerato, en el período de 1995-2003 son 109 (29.6\%). Las investigaciones y tesis de Pedagogía han privilegiado de manera preponderante el campo o nivel de la Educación Media o Educación Secundaria con un total de 93 tesis (20.6\%), a los que habría que añadir un $6.7 \%$ referidas al Bachillerato, lo que hace un total de 130 tesis (27.3\%); seguido de la Educación General Básica 19\% y de la Primaria, 13.7\% (total 67 tesis); finalmente al nivel Pre-escolar se dedican 19 tesis $(3.8 \%)$. 


\begin{tabular}{|l|r|r|r|}
\hline Niveles & \multicolumn{1}{|c|}{ ISS } & \multicolumn{1}{c|}{ UPS } & Total \\
\hline Prenatal & 0 & 1 & $\mathbf{1}$ \\
\hline Preescolar & 1 & 18 & $\mathbf{1 9}$ \\
\hline Primaria & 6 & 61 & $\mathbf{6 7}$ \\
\hline EGB & 1 & 91 & $\mathbf{9 2}$ \\
\hline Ciclo Básico & 5 & 18 & $\mathbf{2 3}$ \\
\hline Secundaria & 40 & 61 & $\mathbf{1 0 1}$ \\
\hline Bachillerato & 3 & 30 & $\mathbf{3 3}$ \\
\hline Superior & 2 & 3 & $\mathbf{5}$ \\
\hline Formación Docente & 0 & 6 & $\mathbf{6}$ \\
\hline Pastoral Juvenil & 13 & 11 & $\mathbf{2 4}$ \\
\hline Educación Popular & 4 & 4 & $\mathbf{8}$ \\
\hline Educación Adultos & 2 & 5 & $\mathbf{7}$ \\
\hline Artesanal & 0 & 3 & $\mathbf{3}$ \\
\hline Religiosos & 7 & 4 & $\mathbf{1 1}$ \\
\hline Sin información & 38 & 52 & $\mathbf{9 0}$ \\
\hline Total por época & $\mathbf{1 2 2}$ & $\mathbf{3 6 8}$ & $\mathbf{4 9 0}$ \\
\hline
\end{tabular}

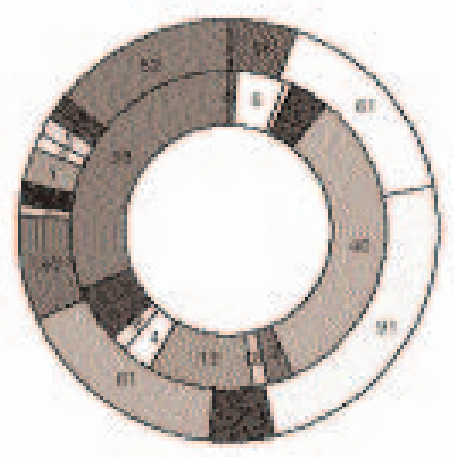


Tres datos resultan muy significativos: a) el elevado número de tesis sin definición del nivel educativo que tiene por objeto: 90 (18.4\%); b) la gran dispersión de tesis que cubren áreas educativas al margen de los niveles de instrucción formal (artesanal, educación de adultos, popular, formación docente): 24 tesis $(4.94 \%)$; c) el número relativamente grande de tesis que no tienen referencia pedagógica a nivel educativo como son las dedicadas a la pastoral juvenil (24:4.9\%) y las religiosas $(11 ; 2.24 \%)$. Este fenómeno se explica en parte por las preferencias de muchos estudiantes procedentes del medio eclesiástico, y para quienes los intereses pastorales y religiosos se sobreponían a los específicamente científicos y académicos de la pedagogía. De acuerdo a información de entrevistas nunca quedó claro para este sector que una buena formación pedagógica podía mejorar sus prácticas pastorales y religiosas.

De estos datos cabe deducir algunas conclusiones: en primer lugar, no resultan claras las orientaciones académicas y profesionales de la carrera de pedagogía dada la diversidad de campos o niveles educativos cubiertos por las investigaciones de las tesis; en segundo lugar, a esta falta de orientación de la carrera habrá que atribuir una deficiente especificidad pedagógica - educativa de la carrera, la cual habría propiciado que otros intereses diferentes a los científicos y académicos propios de la pedagogía condicionaran las investigaciones y tesis. Esto mismo viene a confirmar el elevado número de tesis sin referencia a un campo o nivel educativo.

\section{6. Áreas de los planteles educativos y de las investigaciones}

Aunque el mayor número de investigaciones están referidas al área urbana (239: 48.8\%), es muy significativa la cantidad relativamente elevada de tesis que tienen por campo u objeto el área rural (108:22\%), lo cual puede explicarse no solo por el Programa Cotopaxi, que se inicia en 1995 muy centrado en zonas rurales, sino también en parte por la orientación más social que pedagógica de algunos directores de tesis, de los programas de la carrera y de la misma Universidad. Esto mismo estaría confirmado por la especialización académica y profesional de algunos docentes que han dirigido mayor número de tesis. 


\begin{tabular}{|l|r|r|r|}
\hline Áreas & ISS & UPS & Total \\
\hline Internacional & 1 & 1 & $\mathbf{2}$ \\
\hline Nacional & 3 & 2 & $\mathbf{5}$ \\
\hline Rural & 5 & 103 & $\mathbf{1 0 8}$ \\
\hline Urbana & 42 & 197 & $\mathbf{2 3 9}$ \\
\hline Sin información & 71 & 65 & $\mathbf{1 3 6}$ \\
\hline Total por época & $\mathbf{1 2 2}$ & $\mathbf{3 6 8}$ & $\mathbf{4 9 0}$ \\
\hline
\end{tabular}
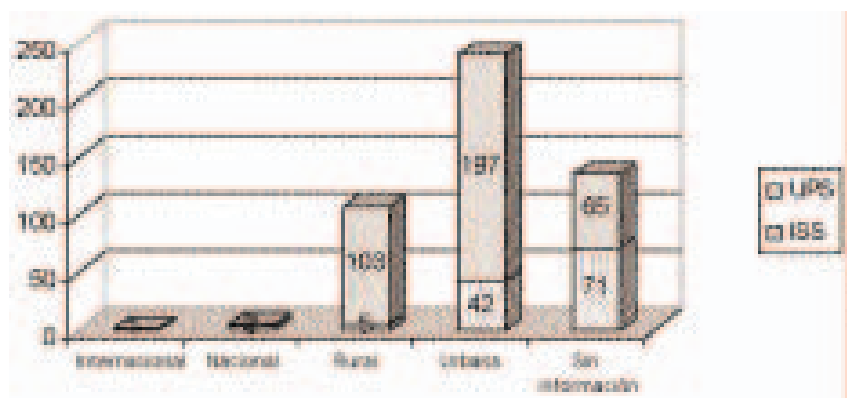

En términos más precisos hay que notar que solo 5 tesis $(4.1 \%)$ corresponden a investigaciones en el área rural entre 1965 y 1994, a diferencia de 42 tesis correspondientes al área urbana (34.4\%), mientras en el período 1995-2003 las investigaciones en el área rural alcanzan el $28 \%$ y la urbana el $53.5 \%$. Pero más ilustrativo es el cambio que se opera respecto del número de investigaciones sin referencia ("sin información") a un área determinada: pasan del 58.2\% en el período 1965-94 al 17.7\% en 1995-2003; lo que supone una mayor definición en las orientaciones de la investigación pedagógica.

7. Régimen de los planteles educativos objeto de las investigaciones

\begin{tabular}{|l|r|r|r|}
\hline SOSTENIMIENTO & \multicolumn{1}{|c|}{ ISS } & UPS & Total \\
\hline Fiscal & 5 & 123 & $\mathbf{1 2 8}$ \\
\hline Particular & 17 & 61 & $\mathbf{7 8}$ \\
\hline Fiscal - Particular & 3 & 15 & $\mathbf{1 8}$ \\
\hline Sin información & 97 & 169 & $\mathbf{2 6 6}$ \\
\hline $\mathbf{N}^{\mathbf{0}}$ Tesis por año & $\mathbf{1 2 2}$ & $\mathbf{3 6 8}$ & $\mathbf{4 9 0}$ \\
\hline
\end{tabular}




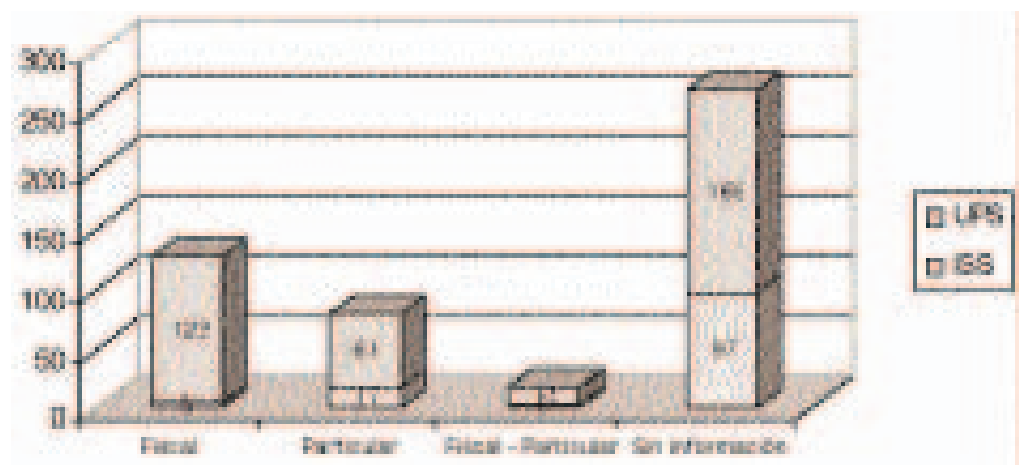

La gran mayoría de las investigaciones se han realizado en planteles educativos de régimen fiscal $(128 ; 26.1 \%)$, seguido de planteles de régimen privado $(78 ; 15.9 \%)$ y de régimen mixto $(18 ; 3.6 \%)$. Un número muy elevado de tesis $(266 ; 54.3 \%)$ carece de referencia sobre el régimen de plantel educativo, ya que no siempre resulta fácil su identificación. Aunque la mayor parte de este grupo de investigaciones no son propiamente pedagógicas y por consiguiente tampoco tienen una referencia a instituciones educativas.

Un cambio muy significativo se opera entre 1965-94, durante el período de formación académica de la carrera de pedagogía, cuando el $13.9 \%$ de las tesis son dedicadas al sector privado de la educación, y el $4.1 \%$ al fiscal; mientras que en la fase de consolidación académica de la carrera, 1995-2003, el 33.4\% de las investigaciones se refieren al sector fiscal y menos de la mitad, $16.6 \%$ al privado. También se nota un cambio muy sensible sobre la orientación específicamente institucional y educativa de las investigaciones, al observarse la diferencia entre los dos períodos de las tesis "sin información"; 79.5\% (1965-1994) frente al 45.9\% (1996-2003). Estos cambios coinciden con los operados en otros aspectos o cualidades de las investigaciones entre los dos períodos. 


\section{Conclusiones y recomendaciones para la investigación y dirección de tesis}

1. Los "currículos" o programas de materias de la carrera deberán organizarse de tal manera que: a) respondan fielmente y de modo operativo a los perfiles académicos y profesionales definidos por la misma programación de la carrera; b) estén bien definidas las áreas de especialización y aplicación científicas y profesionales en el "currículo" de la carrera, delimitando así los campos científico-académicos y profesionales (áreas temáticas) en los que se realizarán las investigaciones o tesis de licenciatura.

1a. Hay que tener en cuenta que una ciencia así como la organización académica de una carrera se desarrollan por un doble proceso de complejización y especialización, al que corresponde siempre una creciente complejización y especialización profesionales. De ahí que parte de la formación científica, académica y profesional del estudiante sea precisamente esta experiencia de especialización. Muy importante para sus futuras competencias y rendimientos profesionales ${ }^{2}$.

1b. Es importante que el trabajo académico de la investigación -tesis articule el componente científico (área de aplicación del conocimiento) con el objetivo de la profesionalización del estudiante, de tal manera que tenga una experiencia profesional de utilización y aplicación de la ciencia, que mejor le prepare para su futuro desempeño laboral. En este sentido urge destacar que la investigación -tesis no es solamente un ejercicio científico y académico sino también profesional, ya que solo aprendiendo a plantear bien los problemas puede un profesional en su campo resolver los problemas propios de su profesión: únicamente un problema bien planteado puede ser bien resuelto. Según esto la investigación tesis constituye el espacio privilegiado donde se articula la formación científica y profesional del estudiante.

2 La misma estructura del mercado laboral y profesional en la sociedad moderna se transforma de manera cada más innovando demandas y de acuerdo a una creciente especialización y complejización.

144 
1c. De acuerdo a los análisis realizados en el transcurso de la investigación es importante advertir sobre aquellas tesis realizadas en base a los llamados PRODUCTOS. Tales trabajos y ejercicios realizados en las ciencias que no son aplicadas, si no se los realiza como parte aplicada de un marco investigativo previo, carecen de valor científico, y tal como suelen realizarse no contribuyen a la formación académica y profesional del estudiante, y mucho menos representan un aporte científico. Un producto es siempre resultado de un desarrollo científico, de la solución de un problema, y en tal sentido la tesis que tiene por objetivo un producto, debería ser el resultado de una investigación previa, y por consiguiente enmarcarse en el mismo contexto académico de las Áreas de conocimiento del currículo y de los Talleres, y sujeto a la misma dirección de tesis que las otras investigaciones.

2. Para garantizar la calidad científica y académica de las investigaciones -tesis por parte de los estudiantes y su dirección por parte de los profesores responsables se requiere que: a) el "currículo" programe 4 o al menos 3 semestres- niveles de TALLER DE TESIS, en el transcurso de los cuales los estudiantes que trabajan en un mismo campo científico o área del conocimiento al mismo tiempo que comparten una formación especializada común, irán definiendo cada uno de ellos el objeto-problema de su propia investigación; b) mientras que los 2 primeros niveles del TALLER DE TESIS constituyen un espacio académico colectivo, donde se conforma ya una experiencia de comunidad científica y académica, en la que se comparten todas las fases del proceso de investigación, en los 2 niveles finales del TALLER DE TESIS se puede ir adoptando una forma de TUTORÍA, donde el director apoya y supervisa cada una de las investigaciones de acuerdo a los particulares desarrollos.

2a. Los TALLERES DE TESIS podrían contar eventualmente con el soporte de algunos SEMINARIOS, para que estos con el tratamiento de temas o problemas afines a los talleres sirvieran de soporte. Por ejemplo, un Taller de sociología rural podría beneficiarse mucho contar con un Seminario de antropología, y a un taller de sociología urbana con un taller sobre violencias o sobre infancia y adolescencia. Esto implica que las Investigaciones -Tesis- de los estudiantes y su dirección por los docentes encargados no se definen por TEMAS sino por ÁREAS del conocimiento, de manera que sean estas el criterio que agrupa a los 
alumnos con sus distintos TEMAS, pero pertenecientes a un mismo CAMPO o ÁREA de conocimiento, así como hace de uno o dos docentes responsables de dichos CAMPOS-AREAS y TALLERES. Lo que no solo permite rentabilizar el recurso humano sino también contribuir a su especialización y mayores competencias académicas y científicas ${ }^{3}$.

2b. Los talleres de tesis tienen la doble ventaja de: a) proporcionar al estudiante una diversificación de los espacios académicos, diferentes de la clase magistral, donde las iniciativas y creatividad del estudiante son más promovidas; b) garantizar que la investigación y la tesis puedan concluirse dentro del ciclo universitario previsto por la carrera, evitando que tan elevado número de egresados nunca lleguen a titularse o se titules deficientemente por no haber terminado su tesis en el plazo de sus estudios.

2c. Son estas ÁREAS de conocimiento, de desarrollo y de aplicación de la ciencia, las que en el currículo de la carrera configuran los espacios académicos de los TALLERES de Investigación-tesis, en los que quedarán enmarcada una triple articulación: científica, académica y profesional. De este modo el PROGRAMA DE INVESTIGACIONES de una carrera coincide, al menos en gran medida, con las ÁREAS de conocimiento y los TALLERES en los que se realizarán las investigaciones y tesis de los estudiantes. Puesto que los criterios para definir aquel Programa son los mismos para definir estos (actuales desarrollos y agendas de una ciencia, intereses y necesidades sociales, desarrollo académico de la Universidad).

3. Esta organización académica de los talleres de tesis e Investigaciones de una carrera tiene efectos de extraordinario valor en diferentes ámbitos:

\footnotetext{
${ }^{3}$ Debido a que los Talleres constituyen un espacio académico poco conocido y peor maltratado, será necesario una cierta capacitación de docentes, que sean directores de tesis, para que desarrollen esta actividad con el mejor uso de dicho espacio académico. Lo que puede hacerse en base a instructivos y encuentros de capacitación. Sobre la organización y prácticas académicas en TALLERES y SEMINARIOS, y su funcionamiento, puede consultarse el libro Docencia Universitaria, cap. 8, dedicado a "variables geometrías académicas de la docencia Universitaria: el seminario y los talleres".
} 
a. Dicho modelo permite a cada carrera desarrollar en el transcurso de un par de años una valiosísima masa crítica sobre un determinado campo de conocimientos, lo que a su vez constituye un aporte muy coherente tanto para el desarrollo académico de la Universidad (para mejorar la docencia, los programas de la carrera o nuevas carreras o postgrados...) como para responder a necesidades e intereses de la sociedad.

b. Refuerza y mejora los vínculos generacionales al asegurar una reproducción científico-académica entre el docente y el estudiante de mayor provecho y rendimiento que el producido únicamente por las clases magistrales.

c. Se hace de la Investigación-tesis no un simple trámite académico- el espacio privilegiado en el que además de articularse la formación científica y profesional es el mismo estudiante el que la completa de manera más personal.

d. Los talleres-tesis contribuyen a una importante especialización del grupo docente, que dirige tesis en un determinado campo de conocimientos, al mismo tiempo que se capacitan para desarrollar ellos mismos sus competencias como investigadores. Esto mismo implica una política académica, que combine una relativa estabilidad de docentes directores de tesis y su dedicación a un determinado campo de conocimientos o Talleres con determinados cambios de nuevos directores y desplazamientos a nuevos campos de conocimiento, para ampliar y mejorar los recursos académicos de la carrera y de la Universidad.

e. Las posibilidades y usos académicos de los talleres y de las investigaciones-tesis son muchos y diversos. Entre otros se pueden aprovechar para preparar las futuras promociones de estudiantes, organizando talleres de talleres, en los que los estudiantes y directores de las tesis-investigaciones presentan sus avances a sus resultados a los estudiantes de los niveles inferiores, con la finalidad de prepararlos o promover sus propios campos de especialización.

f. Es importante tener muy en cuenta el sistema de créditos que merece un TALLER de tesis y su dirección. No puede ser homologable con un 
curso, ya que el tipo de dedicación que requiere es mucho mayor. Cumplir con los requerimientos de un TALLER y al mismo tiempo seguir cinco, seis o más materias afectaría el rendimiento tanto del estudiante como del docente director de tesis.

g. Finalmente este ámbito académico se presenta muy propicio para ser mejorado de muchas maneras. Por ejemplo la elaboración de instructivos para la Dirección de Tesis o para la Dirección de Talleres. Otro sería la creación de líneas o programas de publicación, con los resultados de investigaciones-tesis bien enmarcadas en un determinado campo de conocimientos, lo que siempre proporciona un valor agregado a las investigaciones particulares. 